\title{
QT Dispersion Monitoring as a Coronary Disturbance Prediction Tool
}

\author{
R González, A Rodríguez, R Almeida \\ Central Institute of Digital Research, Cuba
}

\begin{abstract}
The goal of this paper is to discuss a method to study the QT interval dispersion continuously. This parameter is computed for each ten-second-signal strip studied. The relationship between the $Q T$ dispersion variations and the appearance of cardiac disturbances was analyzed. Forty fifteen-minute healthy ECG and five thirty-minute pathologic stress tests were studied. The QRS complexes were detected and classified as normal or premature according to simple rules, the QRS onset was detected using a nine-point derivative function and the $T$ wave offset was identified with an algorithm that follows the shape of the ECG. The QT dispersion graphic was flat and the mean value was less than $60 \mathrm{~ms}$ for every healthy person. However, for each pathologic ECG the QT interval dispersion was over 74 ms before a pathological stage and the graphic was not flat in the minutes previous to the induced cardiac disturbances.
\end{abstract}

\section{Introduction}

The QT interval represents the ventricular activity in the electrocardiogram (ECG) and it has been studied since the beginning of the last century because of its importance [2, 7]. Recently, the QT interval spatial dispersion has emerged as an important indicator associated to ventricular disturbances in cardiac patients; elevated QT dispersion values has been observed in coronary patients with post infarct complications [1,3].

Typically, the QT interval dispersion has been studied on the twelve-lead rest ECG [1]. It is defined as the maximum difference between the smaller and the larger QT interval duration when the twelve leads are acquired simultaneously $[9,12]$.

The identification of the QRS complex onset and the T wave offset are very important to get a reliable QT dispersion measurement [12]. The $\mathrm{T}$ wave offset is not a well-defined point in the ECG because of the influence of several noise and interference sources such as $60 \mathrm{~Hz}$ interference, baseline wandering, electromyographic noise, etc. The QRS onset is affected by the same noise sources, but it is better defined because the QRS complex is a high-energy zone in the ECG $[6,13]$.

Commonly, the QT spatial dispersion has been studied as follows: a ten-second ECG strip is digitized, an average cardiac cycle is computed and the QT interval duration is measured lead by lead. Finally, a unique value of QT dispersion is computed as the difference between the maximum and minimum QT interval duration $[1,7]$. The authors of this paper propose to study the QT spatial dispersion with time in order to evaluate its behavior. This kind of study could be useful to predict cardiac disturbances in coronary patients. The author's hypothesis is the following: the QT dispersion trend with time should be stronger than the study of a unique QT dispersion value to predict a cardiac complication.

\section{Methods}

The proposed method starts with the ECG analogue to digital conversion process. The independent leads (I, II, V1, V2, V3, V4, V5, V6) are sampled simultaneously at a rate of $500 \mathrm{~Hz}$; leads III, aVR, aVL and aVF are computed according to classic expressions based upon leads I and II [11]. Also, a digital moving-average filter proposed by Ligtenberg and Murat [10] is applied in real time. The filter general expression is the following:

$$
y(k)=\frac{1}{K^{2}} \sum_{m=k-K+1}^{k} \sum_{n=m-K+1}^{m} x(n)-\frac{1}{L^{2}} \sum_{m=k-L+1}^{k} \sum_{n=m-K+1}^{m} x(n)
$$

where:

$\mathrm{x}(\mathrm{n})$ : input signal

$\mathrm{y}(\mathrm{k})$ : filtered signal at $\mathrm{k}$ instant

$\mathrm{K}$, L: filter constants associated to cut-off frequency

This expression was simplified and implemented for real-time conditions. It acts like a bandpass filter and the $\mathrm{K}$ and $\mathrm{L}$ constants are associated to the cut-off frequencies. The band pass was set between 0.6 and 37 $\mathrm{Hz}$ in order to remove electromyographic artifacts and to avoid baseline wander [8].

Forty healthy rest ECG and five pathologic stress tests were studied. The standard rest ECG was digitized and filtered during fifteen minutes for each healthy person; each stress test was a thirty-minute ECG with ischemic signs induced by exercise. All the signals were acquired 
with digital devices that are able to store digital signals. Later, the QT spatial dispersion was computed for each ten-second strip. However, the strips including more than two premature beats were excluded in order to compute a robust average cardiac cycle for each strip. The QT spatial dispersion values were plotted versus time for each studied ECG.

The proposed method to computed the QT spatial dispersion included the following steps:

- QRS complex detection.

- QRS complex classification.

- QRS-T complex averaging.

- QRS onset identification

- T wave offset identification.

The QRS complex detection process was based on the Function of Spatial Velocity (FSV) used by the authors in previous studies $[4,5]$. This function can be described by the following expression:

$$
y(k)=\sum_{i=1}^{C}[x(i, k)-x(i, k+1)]^{2}
$$

where:

$$
\begin{aligned}
& \mathrm{y}(\mathrm{k}) \text { : function of Spatial Velocity } \\
& \mathrm{x}(\mathrm{k}) \text { : input signal } \\
& \text { C: studied leads }
\end{aligned}
$$

The FSV was computed sample by sample and a maximun was identified for each ten-second strip in order to set two thresholds. One threshold set the border between the high-energy peaks, associated to the QRS complexes, and the rest of the signal. The second threshold was used to set the first approach to the real onset and offset for each QRS complex. These points were the elements used to compute the duration of each QRS complex; the candidates to be a true QRS complex should reach a minimum duration. Also, the complex onset and offset were used as borders to identify a fiducial point (absolute maximum value of the signal in lead II) to compute the RR interval duration. It was defined as the distance between two consecutive fiducial points. An average value was computed for the RR interval and QRS complex duration, these values were the basic tools for the QRS classification process.

Each QRS complex detected was classified as normal or premature in order to exclude the premature beats form the average beat computed for each ten-second strip studied. The following sentences were applied to make this kind of classification:

- The duration of a RR interval previous to a normal beat (NB) must be between $80 \%$ and $110 \%$ of the average duration of this interval.
- The duration of a normal beat must be between $85 \%$ and $110 \%$ of the average QRS duration.

The authors have set the above limit values in previous studies $[4,5]$. This step is very important because any premature beat should be considered to compute the average cardiac cycle for each signal strip studied. Also, the classification rules should be effectives and easy to implement because one of the objectives of the proposed method is to be used by bedside monitors in real time.

The next step was to compute an average cycle for each lead. The procedure was as follow:

- The maximum QRS value was set as a reference point (Rpoint).

- The cycle onset was set as the sample placed 60 ms before the Rpoint.

- The cardiac cycle offset was defined as the sample placed at Rpoint plus the $60 \%$ of the RR interval average.

- The average cycle was computed with the normal beats for each lead.

The QRS onset and the T wave offset were identified for each average cycle in order to be able to compute the QT spatial dispersion. A search back was made starting at the Rpoint, it was stopped when the derivative absolute value was less than two during $10 \mathrm{~ms}$. A nine-point derivate function was used in this process to avoid the influence of high-frequency noise [13]. The derivative function has the following expression:

$$
y(n T)=\sum_{k=-4}^{k=4} k x(n T+k)
$$

where:

$y(n T)$ : derivate value for each sample. $\mathrm{x}(\mathrm{nT})$ : input signal.

The T-wave onset is not a well-defined event in the ECG. The identification of this point is difficult because it is in a low frequency and noisy zone [12]. Also, it is impossible to associate this point to an important change of slope or polarity. Many efforts have been developed to this topic, but a robust and efficient solution for this problem is not available yet $[1,12]$.

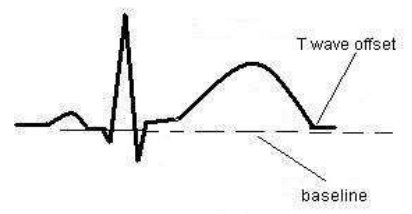

Figure 1. $\mathrm{T}$ wave offset and baseline. 
An algorithm was developed to detect the $\mathrm{T}$ wave offset. This algorithm follows the ECG shape looking for the minimum distance between the signal and the estimated baseline. The developed algorithm was divided in the following steps:

1. Baseline was estimated as the average of the samples associated to the $20 \mathrm{~ms}$ previous to the QRS onset.

2. T wave peak identification: The ST segment end was identified using an algorithm developed by the authors [4]. This point was the beginning of a search window to find the $\mathrm{T}$ wave peak as the absolute maximum value of the ECG in this window. A proportional relationship between the RR interval and QT interval durations was used to set the width of the search window.

3. Looking for the end at the $T$ wave second branch: The absolute electrical difference with the estimated baseline was computed sample by sample until the difference was not decreasing by $10 \mathrm{~ms}$.

Finally, the QT interval duration was computed as the difference in milliseconds between the $\mathrm{T}$ wave offset and the QRS complex onset. The QT dispersion was the difference in duration between the longest QT interval in any lead and the shortest. It was computed for each tensecond ECG strip studied.

\section{Results}

Three thousand six hundred ten-second strips from healthy ECGs were studied. Also, 655 strips from pathological stress tests were analyzed. The proposed method was tested by two highly-qualified cardiologists that analyzed the ECGs on a personal computer with several visual and graphic tools, but they were not aware of the method results. The opinions and conclusions of the specialists were the golden rule to test the proposed method.

There was a full coincidence between the specialists and the proposed method in the QRS complex detection. False positives and false negatives were absent of the results. The behavior of the proposed method in the QRS classification process can be summarized as follow:

- Premature beats only were identified in the stress tests studied. The specialists detected the same.

- Almost 5\% of normal beats were considered as premature, but a premature beat never was classified as normal.

The specialists, helped by a graphic interface program, could evaluate the reliability of the QT interval border points identified by the proposed method. The QRS onset and the $\mathrm{T}$ wave offset were tested; beat by beat, in fifty ten-second ECG strips selected randomly from the healthy ECGs and the same quantity from the stress tests. The main results were the following:

- The maximum difference between the QRS onsets detected by the proposed method and the specialists was $6 \mathrm{~ms}$ (three samples).

- The maximum difference between the $\mathrm{T}$ wave offsets detected by the proposed method and the specialists was $14 \mathrm{~ms}$ (seven samples). This maximum difference was reached in three ECGs characterized by low-voltage $\mathrm{T}$ waves (less than $0.2 \mathrm{mV})$.

Table 1

Minimum, average and maximum QT dispersion values for each case studied

\begin{tabular}{|c|c|c|c|c|c|c|c|}
\hline Case & Min. & Ave. & Max. & Case & Min. & Ave. & Max. \\
\hline N1 & 28 & 34 & 46 & N24 & 34 & 51 & 68 \\
\hline N2 & 30 & 39 & 48 & N25 & 22 & 29 & 32 \\
\hline N3 & 36 & 39 & 42 & N26 & 26 & 32 & 46 \\
\hline N4 & 28 & 34 & 50 & N27 & 34 & 40 & 48 \\
\hline N5 & 34 & 39 & 42 & N28 & 36 & 45 & 74 \\
\hline N6 & 26 & 33 & 42 & N29 & 30 & 44 & 48 \\
\hline N7 & 28 & 37 & 44 & N30 & 30 & 35 & 48 \\
\hline N8 & 34 & 41 & 48 & N31 & 32 & 39 & 46 \\
\hline N9 & 28 & 36 & 48 & N32 & 44 & 48 & 52 \\
\hline N10 & 30 & 43 & 46 & N33 & 42 & 47 & 50 \\
\hline N11 & 26 & 43 & 48 & N34 & 36 & 44 & 50 \\
\hline N12 & 32 & 45 & 52 & N35 & 30 & 43 & 48 \\
\hline N13 & 28 & 37 & 44 & N36 & 38 & 44 & 50 \\
\hline N14 & 34 & 41 & 48 & N37 & 40 & 45 & 48 \\
\hline N15 & 28 & 36 & 48 & N38 & 32 & 38 & 42 \\
\hline N16 & 30 & 43 & 46 & N39 & 36 & 41 & 44 \\
\hline N17 & 26 & 43 & 48 & N40 & 30 & 47 & 50 \\
\hline N18 & 32 & 45 & 52 & ST1 & 40 & 63 & 74 \\
\hline N19 & 34 & 41 & 48 & ST2 & 48 & 66 & 80 \\
\hline N20 & 28 & 36 & 48 & ST3 & 38 & 58 & 78 \\
\hline N21 & 30 & 43 & 46 & ST4 & 44 & 61 & 86 \\
\hline N22 & 26 & 43 & 48 & ST5 & 48 & 59 & 76 \\
\hline N23 & 32 & 45 & 52 & & & & \\
\hline & & & & & \\
\hline
\end{tabular}

Nn: Normal ECG n. STm: Stress test m. Values in ms

The behavior of the QT spatial dispersion was very similar for all the healthy ECGs. Its average value changed from a patient to another (between 22 and 52 $\mathrm{ms}$ ), but the shape of the dispersion versus time graphic always was flat; only two patients reached isolated values of 68 and $74 \mathrm{~ms}$ each one. A continuous increment of the QT dispersion never was observed in this kind of patients.

The QT spatial dispersion had a different behavior in pathological signals. The value of this parameter reached $86 \mathrm{~ms}$ (a case) and the dispersion was increasing before pathological situations like the increment of the 
ventricular beat rate took place. However, at the end of the stress test, when the patient was resting, the QT dispersion returned to values below $60 \mathrm{~ms}$, but its values were not stables.

\section{Discussion and conclusions}

The results can be classified as good. All the QRS complexes were detected, it was due to the simultaneously acquisition of the twelve leads and the shape of the Function of Spatial Velocity for eight channels. In fact, the same performance was reported by the authors in previous investigations $[4,5]$.

The complex classification had a performance according to the application requirements. The rules used to classify the QRS complexes were simples and very effectives, the main goal was to exclude the premature beats from the average complexes and never this kind of complex was misclassified. A small quantity of normal complexes was misclassified, but this situation did not affect the proposed method performance.

The identification of the QRS complex onset and the T wave end always was effective. The difference between the specialists and the proposed method never was important if a visual observer is considered as a reference. It is important to know that a millimeter is the representation of $40 \mathrm{~ms}$ of signal when the ECG is printed in an ECG machine at $25 \mathrm{~mm} / \mathrm{s}$.

The pathological stress tests studied were the way to analyze the evolution of a patient from a nornal condition to a pathological status provoked by exercise. It is very important because the authors want to know if the study of the QT dispersion across the time can be used as a prediction tool of cardiac complications in coronary patients. The QT dispersion trend in pathological signals was increased in the minutes previous to a cardiac complication and was under $60 \mathrm{~ms}$ when the patient was in a normal status. This fact is very important because the continuous study of QT dispersion could be used as a tool to predcit cardiac complications. When QT dispersion was studied in healthy patients, a very flat dependence with time was observed. The dispersion average values were alway below $52 \mathrm{~ms}$ with only two isolated values over $70 \mathrm{~ms}$ in more that 600 ten-second ECG strips studied.

Two isolated high values of QT dispersion were observed in the healthy ECGs studied. This is an element to consider the study of the QT dispersion trend stronger than the study of isolated values.

The study of the QT dispersion looks like a powerful tool to predict cardiac complications in coronary-care patients. Nevertheless, the size of the studied population should be increased to make a final conclusion.

\section{References}

[1] Batchvarov N, Malik M. Measurement and interpretation of QT dispersion. Program of Cardiovascular Diseases, 2000; 42: 325-344.

[2] Bermúdez F. Electrocardiografía Diagnóstica, Editoral Mc Graw Hill Interamericana de Venezuela, 1998

[3] Ellinor PT, Milan DJ, MacRae CA. Risk stratification in the long-QT syndrome. New England Journal of Medicine. 2003; 349(9):908-9

[4] González R. A Spatial Study of the ST Segment. Proc. Eng. in Med. and Biol. Soc. 25th Annual Intern. Conf., Cancún México, 2003, pp. 2610-2614

[5] González R, Fernàndez R, Raola MC. Real-Time QT Interval Measurement. World Congress on Medical Physics and Biomedical Engineering. Chicago, 2000.

[6] Grigioni F, Boriani G, Magelli C, Branzi A. QRS interval time-related changes and prognosis in heart failure. American Journal of Cardiology. 2003 Feb 15; 91(4):514.

[7] Huang CH, Chen WJ, Chang WT, Yip PK, Lee YT. QTc dispersion as a prognostic factor in intracerebral hemorrhage. American Journal of Emergency Medicine. 2004 May; 22(3):141-4.

[8] International Standard IEC 60601-2-51, Medical electrical equipment - Part 2-51: "Particular requirements for safety, including essential performance, of recording and analyzing single and multichannel electrocardiographs", First Edition 2003-02

[9] Kligfield P, Okin PM, Lee KW, Dower GE. Significance of QT dispersion. American Journal of Cardiology. 2003 May 15; 91(10):1291.

[10] Ligtenberg A, Murat K. A Robust Digital QRS Detection Algorithm for Arrythmia Monitoring. Computer and Biomedical Research; 1983; 16: 273-86.

[11] Macfarlane PW, Macfarlane DK, Podolski M. Mingocare: A new program for automated electrocardiogram interpretation. Electromédica; 1984; 52: 57-61.

[12] Malik M., Batchvarov VN. Measurement, interpretation and clinical potential of QT dispersion. Journal of the American College of Cardiology, 2000; 36:1749-1766.

[13] Tompkins WJ. Biomedical Digital Signal Processing. PTR. Prentice-Hall. 1993.

Address for correspondence

René González Fernández

Calle 202 \#1704 entre 17 y 19, Playa. La Habana, Cuba. rigonzalez@icid.cu 